\title{
Challenges in Staging Systems for Colorectal Cancer: Clinical Significance of Metastatic Lymph Node Number in Colorectal Cancer and Mesorectal Extension in Rectal Cancer
}

\author{
Yoshito Akagi Takanaru Fukushima Tomoaki Mizobe Ichitaro Shiratsuchi \\ Yasuhiko Ryu Takefumi Yoshida Nobuya Ishibashi Tetsushi Kinugasa \\ Kazuo Shirouzu
}

Department of Surgery, Kurume University, Kurume, Japan

\section{Key Words}

Staging system for colorectal cancer $\cdot$ Number of metastatic lymph nodes $\cdot$ Mesorectal extension

\begin{abstract}
In many countries, treatment for cancer is performed based on staging systems in which the degree of cancer development is defined objectively. A common staging system is thus needed to compare outcomes. The staging system for colorectal cancer in Japan has been made to enhance consistency with the TNM classification, and the categorization of metastatic lymph nodes and depth of invasion have been revised in recent years. Although these are important factors that determine disease stage, relationships between each factor and recurrence have shown differing prognoses. In our retrospective study, the prognosis of a group with only one metastatic lymph node was significantly better compared to a group with $\geq 2$ metastatic lymph nodes. In addition, rectal cancer with mesorectal extension $>5 \mathrm{~mm}$ showed low relapse-free survival rates and high recurrence rates. The validity of staging systems should thus be inspected from various perspectives.

Copyright $\odot 2010$ S. Karger AG, Basel
\end{abstract}

\section{Introduction}

Predicting prognosis and determining guidelines for therapeutic strategies is important in patients with carcinoma. A staging system that can objectively express the degree of carcinoma development is thus necessary. Around the world, various well-known staging systems are used to reflect prognosis for colorectal cancer such as Dukes classification [1], the Astler Coller classification [2] and TNM classification [3]. Moreover, original staging systems are used in other countries, such as the UK and Australia.

In Japan, original clinical studies on colorectal cancer have been repeated and general rules for clinical and pathological studies of cancer of the colon, rectum and anus have been modified and applied on the basis of those results [4]. The important prognostic factors for colorectal cancer are depth of invasion and regional lymph node metastasis, and these factors are used to determine disease stage [1, 5-7]. However, definitions of the degree of metastasis to lymph nodes and depth of invasion vary slightly between each staging system. A standard staging system allowing the comparison of procedure is necessary if the globalization of cancer therapy is to advance. 
From this perspective, in the 7th Japanese edition of the general rules for clinical and pathological studies on cancer of the colon, rectum and anus, considerable changes have been made to enhance consistency with both the TNM classification and Japanese classifications of other gastrointestinal cancers [8]. Classification of lymph nodes in our country has been grouped in part in a principal bronchus artery such as the inferior mesenteric artery. The pericolic nodes along the marginal arteries were classified as $\mathrm{N} 1$, the lymph nodes at the pedicle of the principal vessel chosen were classified as N3, and the lymph nodes between $\mathrm{N} 1$ and $\mathrm{N} 2$ were classified as $\mathrm{N} 2$.

In terms of the grading of lymph node metastasis, classification by both grouping which was mentioned above and the number of regional lymph node metastases was considered from the classification by grouping alone [8]. However, conventional classification by grouping appears to reflect the prognosis in some groups. Furthermore, in the TNM classification, $\leq 3$ metastases to lymph nodes are provided as N1 and $\geq 4$ as N2. However, doubt remains about the basis and validity of this cut-off.

Regarding depth of invasion, some problems remain in the diagnosis of extramural infiltration in parts without serosa. In the Japanese Classification of Colorectal Carcinoma (JCCRC), tumor invasion infiltrating through the muscularis propria in the part without serosa is classified as slight invasion (A1) or deep invasion (A2). However, this definition is vague and no prognostic difference between these categories has been shown. Given these facts, the classification of tumor invasion has been revised so that tumor invasion through the muscularis propria (T3 in the TNM classification) is represented by ' $\mathrm{A}$ ' in Japan [8]. The degree of invasion has been suggested to correlate with prognosis, so there seems to be room for examination of this point.

The present study investigated relationships between the degree of lymph nodes metastasis, extent of tumor invasion and prognosis. We then reviewed an outline of the significance of these relationships.

\section{Materials and Methods}

\section{Metastatic Lymph Node (MLN) Patients}

From 1975 to 2000, a total of 462 consecutive patients with Dukes $\mathrm{C}$ colon cancer treated by curative resection were collected from the colorectal cancer database of Kurume University, Fukuoka, Japan. The median age of these patients was 62 years, and 290 patients were male (62.7\%). Colon lesions were present in 215 patients, with rectal lesions in 247. The median number of retrieved lymph nodes was 29 (range $28-50$, average $32.2 \pm 18.1$ ), and median duration of follow-up was 68 months (range 24-186, average $60.5 \pm 22.3$ ). These cases were classified by the number of MLNs and the survival rate of each group was determined. Then a new appropriate classification was considered from the number of cases and survival rate. In a new classification, a survival rate was reviewed again and prognostic-related factors were inferred statistically.

\section{Mesorectal Extension (ME) Patients}

From 1982 to 1990, a total of 220 consecutive patients with rectal cancer (Dukes B, $\mathrm{n}=114$; Dukes $\mathrm{C}, \mathrm{n}=106$ ) treated by curative resection were collected from the colorectal cancer database of Kurume University, Fukuoka, Japan. Patients who received preoperative radiotherapy were excluded. Overall median follow-up was 75 months (range 6-198). The endpoint for survival was the time at recurrence of rectal cancer. Relationships between recurrence and distance of ME and other clinicopathological factors were analyzed statistically.

\section{Measurement of $M E$}

Measurement of ME was performed as follows. When the layer of muscularis propria was completely torn, the distance perpendicular to the straight line between both inferior margins of the torn muscularis was measured (fig. 1a). When the continuity of the muscularis propria layer was unclear, the judgment of the rift was assumed of the degree of tearing and the distance from the muscularis propria to the deepest part of the carcinoma was measured (fig. 1b).

\section{Statistical Methods}

Analysis of variance or a t test were used to analyze continuous variables. A $\chi^{2}$ approval was used for categorical variables. Fiveyear survival rates and prognostic factors were estimated using the Kaplan-Meier survival method and Cox proportional hazard regression model, respectively. A multivariate statistical study using Cox proportional hazard modeling was applied to identify independent variables influencing survival. Values of $\mathrm{p}<0.05$ were considered statistically significant. Statistical analysis was performed using StatView 5.0 software (SAS Institute Inc., Cary, N.C., USA).

\section{Results}

\section{MLN Analysis}

Survival rates were compared for groups classified by the number of MLNs. The 5-year survival rate (number of cases, median number of retrieved lymph nodes) for each group were as follows, respectively: $1 \mathrm{MLN}, 80.2 \%$ (163 cases, 28 nodes); 2 MLNs, 68.1\% (83, 29); 3 MLNs, $69.2 \%$ (64, 31); 4 MLNs, $71.7 \%$ (49, 34); 5 MLNs, 68.1\% (26, 33); 6 MLNs, $68.6 \%(19,43) ; 7$ MLNs, $59.3 \%(11,36) ; 8$ MLNs, $49.7 \%(12,41) ; 9$ MLNs, $32.7 \%(10,48)$, and $\geq 10$ MLNs, $29.9 \%(25,50)$. Thus the 5 -year survival rate for the group with 1 MLN was significantly better than the other groups. And the group with $\geq 7$ MLNs showed a 
Fig. 1. Method of measurement of mesorectal extension (ME). a Muscularis propria (MP) completely and clearly torn. b Unclear status of MP. * MP bottom line between both inferior margins. ${ }^{* *}$ Tumor bottom line.

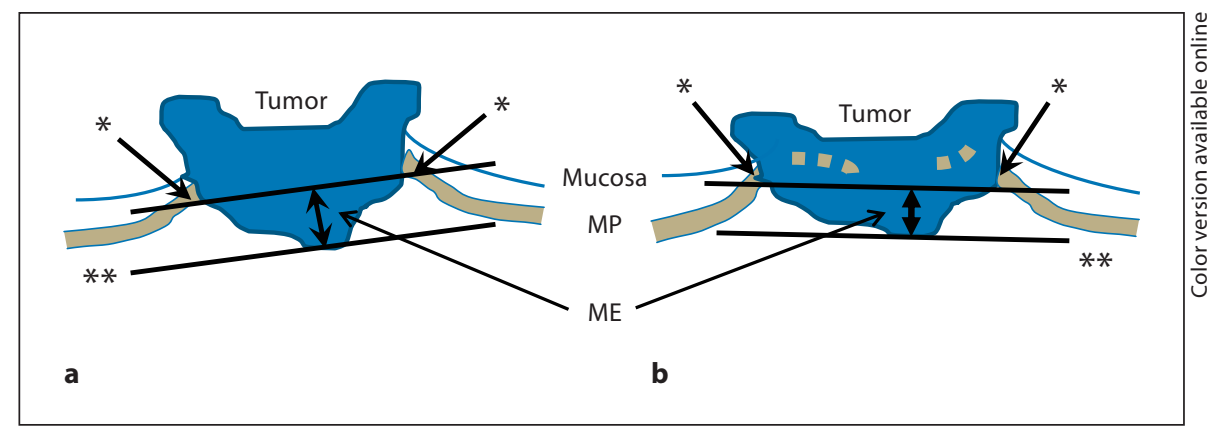

significantly lower survival than groups with $\leq 6$ MLNs. Based on the above-mentioned results, the survival curve of each group (data not shown), survival rate, patient number and current classification were considered and integrated and reorganized into five groups.

The new classification used 1 MLN for group A (163 cases), 2 MLNs for group B (83 cases), 3 MLNs for group C (64 cases), 4-6 MLNs for group D (94 cases), and $>6$ MLNs for group E (58 cases). The 5-year survival rate and relapse-free survival rate for each group were: $A, 80.2$ and 75.5\%; B, 67.0 and $62.0 \%$;, 66.3 and $61.9 \%$; D, 66.8 and $61.9 \%$, and E, 39.6 and $39.6 \%$, respectively. The survival curves of groups B-D were similar (fig. 2). This classification such as group A, groups B-D and group E was thus more capable than conventional categories such as groups A-C and groups D-E. In brief, MLN categories seemed the most adequate when the number of metastases was classified as $1,2-6$, or $\geq 7$. Factors influencing prognosis were statistically extracted from clinicopathological factors of every group. Furthermore, correlative prognostic factors included in the new classification of MLN degree were estimated with uni- and multivariable analyses (table 1). As for independent prognostic factors, preoperative carcinoembryonic antigen level, degree of vessel invasion, and MLN number were extracted.

\section{ME Analysis}

Median ME was $3.8 \mathrm{~mm}$ (range $0.2-30$, average 4.9 \pm 4.3). The cut-off value of ME was determined as follows. Measured ME values were divided every $1 \mathrm{~mm}$ and classified into two groups (e.g., $<1$ vs. $\geq 1 \mathrm{~mm},<2$ vs. $\geq 2 \mathrm{~mm},<3$ vs. $\geq 3 \mathrm{~mm},<4$ vs. $\geq 4 \mathrm{~mm}, \ldots$.). These groups were compared with disease-free survival respectively and each $p$ value was calculated. The cut-off value was fixed for the ME value associated with the lowest $\mathrm{p}$ value by comparison between the above two groups, and was considered as a factor influencing recurrence.

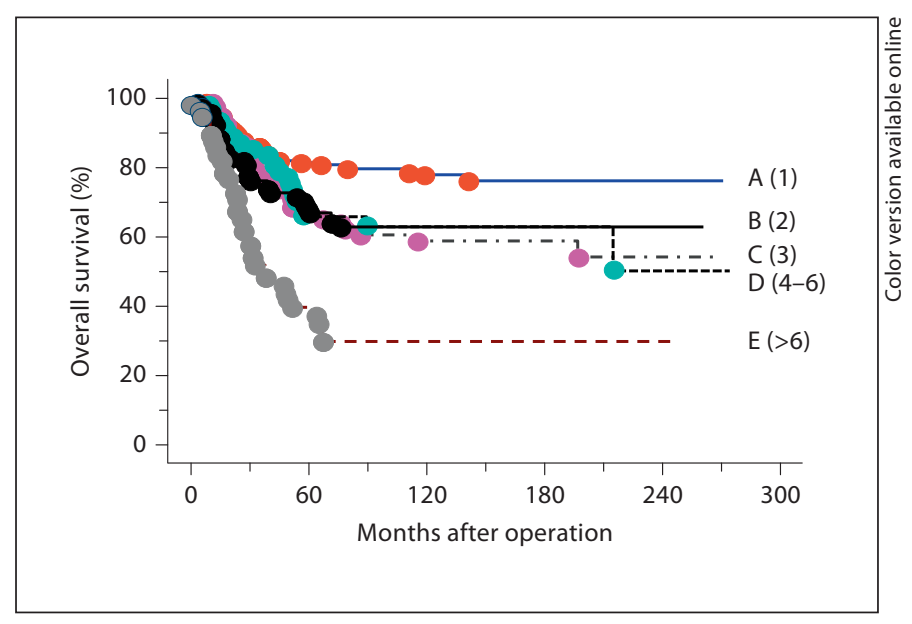

Fig. 2. Survival curve according to the number of metastatic nodes.

When the cut-off value for ME was fixed at $5 \mathrm{~mm}$ for rectal cancer, the $\mathrm{p}$ value by comparison of relapse-free survival between groups ( $<5 \mathrm{vs}$. $\geq 5 \mathrm{~mm})$ was lowest. Clinicopathological factors related to recurrence of rectal cancer were location (upper/lower), operative procedure (anal preservation/no preservation), tumor gross type (localized type/invasive type), depth of invasion (a1/a2), histologic type (well/others), lymphatic invasion (negative/positive), MLNs (negative/positive) and pelvic lymph node metastasis (negative/positive), according to univariate analysis. Adding $\mathrm{ME}(<5 / \geq 5 \mathrm{~mm})$ to these factors, multivariate analysis was performed, sphincter-preserving operation (yes/no), depth of invasion (a1/a2), lymphatic invasion (negative/positive), MLNs (negative/positive), pelvic lymph node metastasis (negative/positive) and actual measurement $\mathrm{ME}(<5 / \geq 5 \mathrm{~mm})$ were extracted as independent risk factors for recurrence (table 2). 
Table 1. Multivariate analysis of independent prognostic factors

\begin{tabular}{llll}
\hline Variable & $\mathrm{p}$ & Hazard ratio & 95\% CI \\
\hline Preoperative CEA level $(<5.0$ vs. $\geq 5.0)$ & 0.0075 & 1.508 & $1.069-2.123$ \\
Vessel invasion (negative vs. positive) & 0.0140 & 1.466 & $1.026-2.096$ \\
MLN number (1 vs. $\geq 2$ ) & 0.0332 & 1.783 & $1.087-2.915$ \\
MLN number ( $\leq 6$ vs. $\geq 7)$ & 0.0004 & 2.169 & $1.332-3.534$ \\
\hline
\end{tabular}

$\mathrm{CEA}=$ Carcinoembryonic antigen; $\mathrm{MLN}=$ metastatic lymph nodes .

Table 2. Multivariate analysis of risk factors related to a recurrence

\begin{tabular}{lllll}
\hline Variable & $\chi^{2}$ & $\mathrm{p}$ & Hazard ratio & 95\% CI \\
\hline Sphincter-preserving operation (yes/no) & 23.678 & $<0.0001$ & 1.872 & $1.454-2.410$ \\
Depth of invasion (a1/a2) & 21.546 & $<0.0001$ & 1.817 & $1.412-2.338$ \\
Lymphatic invasion (negative/positive) & 26.538 & $<0.0001$ & 1.876 & $1.876-4.062$ \\
MLN (negative/positive) & 78.637 & $<0.0001$ & 3.403 & $2.596-4.461$ \\
MLN of lateral pelvis (negative/positive) & 67.860 & $<0.0001$ & 6.830 & $4.324-10.788$ \\
Actual measurement of ME $(<5 \mathrm{~mm} / \geq 5 \mathrm{~mm})$ & 25.961 & $<0.0001$ & 1.048 & $1.048-1.110$ \\
\hline
\end{tabular}

MLN $=$ Metastatic lymph nodes; $\mathrm{ME}=$ mesorectal extension.

\section{Discussion}

Grasping cancer progression as a prognostic factor is extremely important when choosing adjuvant chemotherapy and in constructing effective postoperative follow-up systems. Indicators that are strongly associated with prognosis and easily defined are therefore crucial. Lymph node metastasis is an important prognostic factor for colorectal cancer [1, 5-7]. Various studies about relationships between lymph node metastases and prognosis have been reported. Tapper et al. [9] and Nelson et al. [10] mentioned that staging for colorectal cancer requires retrieval of 12-17 lymph nodes. Kim et al. [11] mentioned that retrieval of $>10$ lymph nodes offers almost certain identification of metastasis to lymph nodes, and tumor differentiation and $\mathrm{T}$ stage seem to correlate with higher nodal metastasis rate. Schumacher et al. [12] and Vaccaro et al. [13] recently reported that the lymph node ratio, representing the ratio of the number of MLNs to the number of retrieved lymph nodes, is associated with prognosis. However, whether the classification by the number of MLNs reflects prognosis has yet to be evaluated. For standardization of lymphadenectomy, the extent of lymphadenectomy has been regarded as impor- tant in JCCRC. In Japan, Matsuda et al. [14] recently reported no significant difference between $\mathrm{N} 1$ and $\mathrm{N} 2$ by conventional classification of MLNs. Furthermore, Suzuki et al. [15] also reported that classification by the number of MLNs predicted prognosis better than classification by level of MLNs in node-positive colon cancer. From a retrospective study in our institution, both classification by number of MLNs and level of MLNs revealed a difference in prognosis (data not shown). In the TNM classification the degree of MLNs has used a cutoff of 3 metastatic nodes since the first edition of the classification.

Hermanek et al. [16] reported that the prognosis of colorectal cancer with more than four parabowel MLNs was approximately the same as with MLNs along a principal trunk artery. Since the fifth edition of the TNM classification in 1997, the degree of MLNs was modified disregarding the location of MLNs. Former N3 cases were integrated into $\mathrm{N} 1$ and $\mathrm{N} 2$ subcategories according to the number of MLNs (N1 $\leq 3, \mathrm{~N} 2>3$ ) [17]. Kendal [18] reported that the classification of MLNs was a prognostic factor for rectal cancer, lung cancer and gastric cancer, when the number of MLNs was categorized using a cutoff of 3 metastatic nodes ( $11 \leq 3, \mathrm{~N} 2>3$ ). 
On the other hand, Vaccaro et al. [6] reported that the presence of $>3$ positive nodes was significantly associated with poorer survival rates in uni- and multivariate analyses. As for the data in our institution, the group with only 1 MLN showed a significantly better prognosis than the group with $>1$ MLN. The group with $\geq 7$ MLNs showed a very poor prognosis. These classifications represented an independent prognostic factor in multivariate analysis. It is thought that when several symptom example numbers and progression to rectal cancer were included in the background, metastasis to $>7$ MLNs resulted in a very poor outcome. As more detailed examinations demonstrated approximately similar results, the number of MLNs was suggested to sufficiently reflect a contribution to prognosis. However, the precision of a diagnosis of metastasis to lymph nodes is concerned with personal factors such as range of dissection, dissection maneuvers, and treatment of the specimens. In addition, the number of lymph nodes in the location of the tumor is dependent on the host. Shen et al. [19] also reported the number of lymph nodes obtained in specimens of colorectal cancer as being significantly associated with the length of resected bowel, patient age, and tumor location. There is clearly more room for further examination of MLN degree.

In reports from Europe and America, the cut-off value set by measuring ME for rectal cancer reportedly correlates with recurrence and prognosis [20]. Few reports have concretely described methods of measuring ME [20-22]. Vague phrases such as 'perirectal fat invasion' [23] or 'mesorectal invasion' $[24,25]$ have been used in other reports. Cawthorn et al. [20] reported that in order to set cut-off values of ME to $4 \mathrm{~mm}, 5$-year survival rate must be higher for the group with $\leq 4 \mathrm{~mm}$ in both stage II and stage III. Merkel et al. [26] classified pT3 into pT3a ( $\geq 5 \mathrm{~mm}$ invasion) and $\mathrm{pT} 3 \mathrm{~b}$ ( $<5 \mathrm{~mm}$ invasion), and analyzed data from the Erlangen Registry for Colorectal
Carcinoma and the Study Group for Colorectal Carcinoma. Wittekind et al. [27] reported that the group with $\mathrm{ME}<5 \mathrm{~mm}$ showed higher survival rates in data from the Swiss Registration Study Colorectal Cancer. Miyoshi et al. [21] from Japan reported that ME $>6 \mathrm{~mm}$ is independently associated with poor prognosis in pT3 lower rectal cancer.

Some reports have described the value of ME being associated with local recurrence, although ME was defined in a slightly different manner $[24,26]$. Setting cutoff values for ME may also be achieved by considering overall survival rate $[20,23,26]$, relapse-free survival rate or recurrent rate $[24,25]$. In terms of $\mathrm{pT} 3$ in the TNM classification, subdividing degree of ME into four T categories (pT3a to pT3d) has been suggested to reveal incremental differences [27]. It is clinically important how the degree of ME reflects for staging and whether ME can become a prognostic factor beyond the current staging system.

From some reports, including our own data, measurement of ME may reflect clinically prognostic predictors and judgment of need for adjuvant therapy. Again, this area warrants further investigation. With these results and reports, some problems seem to remain in staging systems for colorectal cancer. The number of MLNs clearly influences the prognosis of colorectal cancer. In addition, in rectal cancer without adventitia, measurement of histological ME is clinically important. Further examinations will help to clarify how these factors can be applied to daily medical treatment.

\section{Disclosure Statement}

The authors declare that no financial or other conflicts of interest exist in relation to the content of the article.

\section{References}

1 Dukes CE, Bussey HJR: The spread of rectal cancer and its effect on prognosis. Br J Cancer 1958;12:309-320.

$\checkmark 2$ Astler VB, Coller FA: The prognostic significance of direct extension of colon and rectum. Ann Surg 1954;139:846-851.

3 Sobin $\mathrm{LH}$, Wittenkind $\mathrm{CH}$ : International Union Against Cancer. TNM Classification of Malignant Tumors, ed 6. New York, Wiley-Liss, 2002.
4 Japanese Society for Cancer of the Colon and Rectum: General Rules for Clinical and Pathological Studies on Cancer of the Colon, Rectum and Anus, ed 1. Tokyo, Kanehara, 1977.

5 Chapuis PH, Dent OF, Fisher R, Newland RC, Pheils MT, Smyth E, Colquhoun K: A multivariate analysis of clinical and pathological variables in prognosis after resection of large bowel cancer. Br J Surg 1985;72:698702 .

\footnotetext{
6 Vaccaro CA, Bonadeo FA, Benati ML, Quintana GM, Rubinstein F, Mullen E, Telenta M, Lastiri JM: Colorectal cancer staging: reappraisal of N/PN classification. Dis Colon Rectum 2004;47:66-69.

7 Choen AM: Prognostic of node-positive colon cancer. Cancer 1991;67:1859-1861.

8 Japanese Society for Cancer of the Colon and Rectum: Japanese Classification of Colorectal Carcinoma, ed 2 English. Tokyo, Kanehara, 2009.
} 
-9 Tapper JE, O’Connel MJ, Niedzwiecki D, et al: Impact of number of nodes retrieved on outcome in patients with rectal cancer. J Clin Oncol 2001;19:157-163.

-10 Nelson H, Pectrelli N, Carlin A, et al: Guidelines 2000 for colon and rectal surgery. J Natl Cancer Inst 2001;93:583-596.

- 11 Kim J, Huynh R, Abraham I, Kim E, Kumar RR: Number of lymph nodes examined and its impact on colorectal cancer staging. Am Surg 2006;72:902-905.

-12 Schumacher P, Dineen S, Barnett C Jr, Fleming J, Anthony T: The metastatic lymph node ratio predicts survival in colon cancer. Am J Surg 2007;194:827-831.

- 13 Vaccaro CA, Im V, Rossi GL, Quintana GO, Benati ML, Perez de Arenaza D, Bonadeo FA: Lymph node ratio as prognosis factor for colon cancer treated by colorectal surgeons. Dis Colon Rectum 2009;52:1244-1250.

14 Matsuda A, Tajiri T, Furukawa K, et al: Relationship between the number of metastatic lymph nodes and the prognosis of colon cancer patients. J Jpn Surg Assoc 2004;65:11711176.
15 Suzuki O, Sekishita Y, Shiono T, Ono K, Fujimori M, Kondo S: Number of lymph node metastases is better predictor of prognosis than level of lymph node metastasis in patients with node-positive colon cancer. J Am Coll Surg 2006;202:732-736.

-16 Hermanek P, Guggenmoos-Holzmann I, Gall FP: Prognostic factors in rectal carcinoma. A contribution to the further development of tumor classification. Dis Colon Rectum 1989;32:593-599.

17 Sobin LH, Wittekind CH: TNM Classification of Malignant Tumors, ed 5. New York, Wiley, 1997.

18 Kendal WS: Lymph node-based prognostics: limitations with individualized cancer treatment. Am J Clin Oncol 2006;29:298-304.

19 Shen SS, Haupt BX, Ro JY, Bailey HR, Schwartz MR: Number of lymph nodes examined and associated clinicopathologic factors in colorectal carcinoma. Arch Pathol Lab Med 2009;133:781-786.

20 Cawthorn SJ, Parums DV, Gibbs NM, et al: Extent of mesorectal spread and involvement of lateral resection margin as prognostic factors after surgery for rectal cancer. Lancet 1990;335:1055-1059.

-21 Miyoshi M, Ueno H, Hashiguchi Y, et al: Extent of mesorectal tumor invasion as a prognostic factor after curative surgery for $\mathrm{T} 3$ rectal cancer patients. Ann Surg 2006;243: 492-498.
22 Katsumata D, Fukui H, Ono Y, et al: Depth of tumor invasion in locally advanced rectal cancer correlates with patients' prognosis: the usefulness of elastic stain for its measurement. Surg Today 2008,38:115-122.

$\checkmark 23$ Picon AI, Moore HG, Sternberg SS, et al: Prognostic significance of depth of gross or microscopic perirectal fat invasion in T3 N0 M0 rectal cancers following sharp mesorectal excision and no adjuvant therapy. Int J Colorectal Dis 2003; 18:487-492.

24 Tokoro T, Okuno K, Hida J, et al: Depth of mesorectal invasion has prognostic significance in T3 N0 low rectal cancer. Hepatogastroenteroloy 2009;56:124-127.

25 Steel MCA, Woods R, Mackay JM, et al: Extent of mesorectal invasion is a prognostic indicator in T3 rectal carcinoma. ANZ J Surg 2002;72:483-487.

26 Merkel S, Mansmann U, Siassi M, et al: The prognostic inhomogeneity in pT3 rectal carcinomas. Int J Colorectal Dis 2001;16:298304.

27 Wittekind C, Greene FL, Henson DE, et al: TNM Supplement: A Commentary on Uniform Use, ed 3. New York, Wiley-Liss, 2003. 